Fixed Point Theory, 20(2019), No. 2, 715-728

DOI: $10.24193 /$ fpt-ro.2019.2.47

http://www.math.ubbcluj.ro/ nodeacj/sfptcj.html

\title{
A NEW CONTRIBUTION TO DISCONTINUITY AT FIXED POINT
}

\author{
N. TAŞ* AND N. YILMAZ ÖZGÜR** \\ *Balıkesir University, Department of Mathematics \\ 10145 Balıkesir, Turkey \\ E-mail: nihaltas@balikesir.edu.tr \\ ** Ballkesir University, Department of Mathematics \\ 10145 Balıkesir, Turkey \\ E-mail: nihal@balikesir.edu.tr
}

\begin{abstract}
The aim of this paper is to obtain new solutions to the open question on the existence of a contractive condition which is strong enough to generate a fixed point but which does not force the map to be continuous at the fixed point. To do this, we use the right-hand side of the classical Rhoades' inequality and the number $M(x, y)$ given in the definition of an $(\alpha, \beta)$-Geraghty type- $I$ rational contractive mapping. Also we give an application of these new results to discontinuous activation functions.
\end{abstract}

Key Words and Phrases: Discontinuity, fixed point, fixed circle, metric space, activation function. 2010 Mathematics Subject Classification: 47H10, 54H25, 47H09.

Acknowledgement. The authors gratefully thank to the referees for the constructive comments and recommendations which definitely help to improve the readability and quality of the paper.

\section{REFERENCES}

[1] R.K. Bisht, R.P. Pant, A remark on discontinuity at fixed point, J. Math. Anal. Appl., 445(2017), 1239-1242.

[2] R.K. Bisht, R.P. Pant, Contractive definitions and discontinuity at fixed point, Appl. Gen. Topol., 18(2017), no. 1, 173-182.

[3] S. Chandok, Some fixed point theorems for $(\alpha, \beta)$-admissible Geraghty type contractive mappings and related results, Math. Sci., 9(2015), 127-135.

[4] L.J. Cromme, Fixed point theorems for discontinuous functions and applications, Nonlinear Anal. Theory, Methods \& Applications, 30(1997), no. 3, 1527-1534.

[5] L.J. Cromme, I. Diener, Fixed point theorems for discontinuous mapping, Math. Program., 51(1991), 257-267.

[6] M. Forti, P. Nistri, Global convergence of neural networks with discontinuous neuron activations, IEEE Trans. Circuits Syst. I, Fundam. Theory Appl., 50(2003), no. 11, 1421-1435.

[7] J. Jachymski, Common fixed point theorems for some families of maps, Indian J. Pure Appl. Math., 25(1994), no. 9, 925-937.

[8] J. Jachymski, Equivalent conditions and Meir-Keeler type theorems, J. Math. Anal. Appl., 194(1995), 293-303. 
[9] R. Kannan, Some results on fixed points, II, Amer. Math. Monthly, 76(1969), 405-408.

[10] X. Nie, W.X. Zheng, On multistability of competitive neural networks with discontinuous activation functions, 4th Australian Control Conference (AUCC), 2014, 245-250.

[11] N. Özdemir, B.B. İskender, N.Y. Özgür, Complex valued neural network with Möbius activation function, Commun. Nonlinear Sci. Numer. Simul., 16(2011), no. 12, 4698-4703.

[12] N.Y. Özgür, N. Taş, Some fixed-circle theorems on metric spaces, Bull. Malays. Math. Sci. Soc., (2017).

[13] N.Y. Özgür, N. Taş, Fixed-circle problem on S-metric spaces with a geometric viewpoint, Facta Univ., Ser. Math. Inf., (accepted).

[14] N.Y. Özgür, N. Taş, U. Çelik, Some fixed-circle results on S-metric spaces, Bull. Math. Anal. Appl., 9(2017), no. 2, 10-23.

[15] R.P. Pant, Discontinuity and fixed points, J. Math. Anal. Appl., 240(1999), 284-289.

[16] B.E. Rhoades, A comparison of various definitions of contractive mappings, Trans. Amer. Math. Soc., 226(1977), 257-290.

[17] B.E. Rhoades, Contractive definitions and continuity, Contemp. Math., 72(1988), 233-245.

[18] M.J. Todd, The Computation of Fixed Points and Applications, Springer-Verlag, Berlin, Heidelberg, New York, 1976.

[19] L.L. Wang, T.P. Chen, Multistability of neural networks with Mexican-hat-type activation functions, IEEE Trans. Neural Netw. Learn. Syst., 23(2012), no. 11, 1816-1826.

[20] Z. Wang, Z. Guo, L. Huang, X. Liu, Dynamical behavior of complex-valued Hopfield neural networks with discontinuous activation functions, Neural Process Lett., 45 (2017), 1039-1061.

Received: May 18, 2017; Accepted: March 9, 2018. 